\title{
Hyperbolic chaotic attractor in amplitude dynamics of coupled self-oscillators with periodic parameter modulation
}

\author{
Isaeva, Olga B.; Kuznetsov, Sergey P.; Mosekilde, Erik
}

Published in:

Physical Review E

Link to article, DOI:

10.1103/PhysRevE.84.016228

Publication date:

2011

Document Version

Publisher's PDF, also known as Version of record

Link back to DTU Orbit

Citation (APA):

Isaeva, O. B., Kuznetsov, S. P., \& Mosekilde, E. (2011). Hyperbolic chaotic attractor in amplitude dynamics of coupled self-oscillators with periodic parameter modulation. Physical Review E, 84(1), - .

https://doi.org/10.1103/PhysRevE.84.016228

\section{General rights}

Copyright and moral rights for the publications made accessible in the public portal are retained by the authors and/or other copyright owners and it is a condition of accessing publications that users recognise and abide by the legal requirements associated with these rights.

- Users may download and print one copy of any publication from the public portal for the purpose of private study or research.

- You may not further distribute the material or use it for any profit-making activity or commercial gain

- You may freely distribute the URL identifying the publication in the public portal 


\title{
Hyperbolic chaotic attractor in amplitude dynamics of coupled self-oscillators with periodic parameter modulation
}

\author{
Olga B. Isaeva, ${ }^{1}$ Sergey P. Kuznetsov, ${ }^{1}$ and Erik Mosekilde ${ }^{2}$ \\ ${ }^{1}$ Kotel'nikov Institute of Radio Engineering and Electronics, Russian Academy of Science, Saratov Branch, \\ Zelenaya 38, Saratov 410019, Russia \\ ${ }^{2}$ Department of Physics, Technical University of Denmark, 28000 Kgs. Lyngby, Denmark
}

(Received 29 April 2011; published 29 July 2011)

\begin{abstract}
The paper proposes an approach to constructing feasible examples of dynamical systems with hyperbolic chaotic attractors based on the successive transfer of excitation between two pairs of self-oscillators that are alternately active. An angular variable that measures the relations of the current amplitudes for the two oscillators of each pair undergoes a transformation in accordance with the expanding circle map during each cycle of the process. We start with equations describing the dynamics in terms of complex or real amplitudes and then examine two models based on van der Pol oscillators. One model corresponds to the situation of equality of natural frequencies of the partial oscillators, and another to a nonresonant ratio of the oscillation frequencies relating to each of the two pairs. Dynamics of all models are illustrated with diagrams indicating the transformation of the angular variables, portraits of attractors, Lyapunov exponents, etc. The uniformly hyperbolic nature of the attractor in the stroboscopic Poincaré map is confirmed for a real-amplitude version of the equations by computations of statistical distribution of angles between stable and unstable manifolds at a representative set of points on the attractor. In other versions of the equations the attractors relate presumably to the partially hyperbolic class.
\end{abstract}

DOI: 10.1103/PhysRevE.84.016228

PACS number(s): 05.45.-a, 05.40.Ca

\section{INTRODUCTION}

Chaotic dynamics in dissipative systems is associated with an object called the strange attractor. Nowadays, the collection of models with strange attractors is very rich, including mathematical examples, as well as models of physical, chemical, and biological systems [1-7].

In the past, mathematical studies focused on a special kind of chaotic attractors: the uniformly hyperbolic attractors. Attractors of this type occur in systems of the so-called axiom A class and are considered in the hyperbolic theory [8-17]. The chaotic nature of dynamics on these attractors is proved rigorously. They possess a property of structural stability: The phase space structure, character of dynamics, and its statistical characteristics are insensitive to variation of parameters and functions in the governing equations. Originally, it was expected that the uniformly hyperbolic attractors might be relevant to many physical situations when dynamical chaos occurs $[11,17,18]$. However, as time passed, it became clear that the multiple known concrete examples of chaos do not fit the narrow frames of the early hyperbolic theory. Therefore, efforts of mathematicians were redirected at generalizations applicable to broader classes of systems $[13,16,19,20]$. Abandoned for a long time and not clarified until recently is the question of the possible occurrence of the dynamical behavior associated with the uniformly hyperbolic attractors in real-world systems (see discussion in Refs. [17,21]). In the theory of oscillations, since the classic works of Andronov and his school, structurally stable (rough) systems have always been regarded as those subjected to priority research, and as the most important for practice $[2,13,22]$. It seems natural that the same should be the case for systems with structurally stable uniformly hyperbolic attractors. The lack of the real-world examples in this regard is an evident dissonance.
Definitions relating to the hyperbolic theory are most easily formulated in the case of the discrete-time dynamics governed by maps (diffeomorphisms). (If needed to deal with continuous time systems, these definitions can be applied using description in terms of a Poincaré map.) An orbit is uniformly hyperbolic if at each point of this orbit in the vector space of all possible infinitesimal perturbations $V$ one can define a subspace of vectors $V_{S}$ decreasing in norm in the evolution forward in time and a subspace of vectors decreasing in reverse time, roughly speaking, exponentially. All vectors in the space $V$ must allow representation as linear combinations of vectors belonging to $V_{S}$ and $V_{U}$ (i.e., the vector space $V$ is a direct sum $V=V_{U} \oplus$ $V_{S}$. The uniformly hyperbolic attractors consist exclusively of orbits of this kind.

Some generalization is partial hyperbolicity $[16,20]$. In this case, the vector space $V$ is a direct sum $V=V_{U} \oplus V_{S} \oplus V_{C}$ of unstable, stable, and center subspaces. The last subspace, $V_{C}$, is associated with perturbations either growing in time more slowly than those relating to $V_{U}$ or decreasing more slowly than those relating to $V_{S}$. Attracting invariant sets satisfying these conditions are called partially hyperbolic attractors.

In textbooks and reviews, examples of the uniformly hyperbolic attractors are traditionally represented by artificial mathematical constructions [8-15,20]. One is the SmaleWilliams solenoid, which can occur in the phase space of maps of dimension 3 or more. Consider a domain in the form of torus and think of it as a plastic doughnut. On one step of the transformation we stretch the doughnut, squeeze it in the transversal direction, fold it to get an $M$-fold loop $(M \geqslant 2)$, and then insert this loop within the original torus. At each next repetition of the procedure, the number of coils is multiplied by the integer $M$, and the total volume of the object decreases (because the map is dissipative). In the limit the number of coils tends to infinity, and volume to zero. In the transversal cross sections the object displays a Cantor-like structure. An obvious 
condition for such an attractor to occur is the presence of an angular variable, that undergoes transformation according to the expanding circle map (or Bernoulli map) $\varphi_{n+1}=M \varphi_{n}$.

The first few examples of feasible continuous-time dynamical systems with attractors of Smale-Williams type in their Poincaré maps were suggested in a number of recent papers by Kuznetsov et al. [23-27]. Here, the role of angular variable was played by the phase of some oscillating process. The systems were composed of two or more oscillators that were activated in alternating manner through transfer of excitation from one oscillator to another with the simultaneous transformation of the phase in accordance with a map of Bernoulli type. The chaotic dynamics revealed itself as a randomlike behavior of the carrier signal in the succession of oscillation bursts generated by the system. Computations based on verification of the so-called cone criterion [10-15] confirmed the hyperbolic nature of the attractors [28] and, for a particular model system considered in Refs. [24,28], an accurate analysis based on the technique of computer-assisted proof has been performed by Wilczak [29].

Due to their structural stability, one expects systems with hyperbolic attractors to be of particular interest as generators of robust chaos for electronic communication systems, random number generators, and various forms of encryption schemes [30-32].

It is worth noticing, however, that the above examples [24-27] describe specially designed devices rather than systems of natural origin. The search for real-world hyperbolic chaotic attractors and demonstration of their possible significance in, e.g., ecology, physiology and neuroscience, or hydrodynamics remains an interesting and challenging problem $[13,21]$. In this perspective it is obviously of interest to broaden our understanding of the type of structures that can be expected to display hyperbolic chaotic attractors.

In this paper we suggest an approach that is based on the amplitude rather than the phase dynamics of the coupled oscillator systems. Section II explains and illustrates the main idea: In a pair of self-oscillators, fed from a common supply, the relative distribution of amplitudes is characterized by an angular variable. We then compose a system of two such pairs of oscillators that alternately are driven into the active regime by means of an external parameter modulation. At the same time, the oscillator pairs are coupled so that excitation is transferred from one pair to the other in such a way that the artificially introduced angular coordinate undergoes multiplication by a factor $M=3$ in each cycle of the external modulation. We demonstrate the hyperbolic character of the observed chaotic attractor through calculation of both the Lyapunov spectrum and the return map over a full modulation period. The return map displays a typical Bernoulli shift character with a slope of 3, and the largest Lyapunov exponent is found to be nearly constant and approximately equal to $\ln 3$.

In Secs. III and IV we examine the implementation of the above approach in two different versions of four coupled van der Pol oscillators. The first example considers the resonant case where the natural frequencies of all four oscillators are equal. The second example, on the other hand, considers a situation in which strong resonances between the two pairs of oscillators do not occur. As believed, in both cases, the attractors are partially hyperbolic: The systems involve (two, respectively one) neutral (or nearly neutral) variables that do not correspond to expanding or contracting directions. However, the presence of these variables apparently does not affect the roughness of the amplitude chaotic dynamics.

\section{AMPLITUDE DYNAMICS IN TERMS OF ANGULAR VARIABLE AND SIMPLE MODEL WITH BERNOULLI-LIKE TRANSFORMATION OF THIS VARIABLE}

Consider first a pair of self-oscillatory elements whose energy losses are compensated from a common source, and suppose the equations for the complex amplitudes $a_{1,2}$ read

$$
\begin{aligned}
& \dot{a}_{1}=\frac{1}{2}\left(1-\left|a_{1}\right|^{2}-\left|a_{2}\right|^{2}\right) a_{1}, \\
& \dot{a}_{2}=\frac{1}{2}\left(1-\left|a_{1}\right|^{2}-\left|a_{2}\right|^{2}\right) a_{2} .
\end{aligned}
$$

The saturation of the amplitudes is determined obviously by a condition $\left|a_{1}\right|^{2}+\left|a_{2}\right|^{2}=1$. At any given time, the current distribution of energy among the oscillators may be characterized by an angular coordinate $\theta$ defined in such way that the amplitudes satisfy the required relation: $\left|a_{1}\right|^{2}=\cos ^{2} \theta$, $\left|a_{2}\right|^{2}=\sin ^{2} \theta$.

Suppose now that we have two such pairs of oscillators, each governed by equations of the type (1), and suppose that they are subjected to periodic parameter modulation produced by same external source in such way that the excitation of the two pairs alternates on successive half periods of the modulation. In addition, we supplement the equations with terms introducing the pairwise coupling between oscillators relating to two alternately active subsystems. The concrete form of these additional terms is selected in such way that the transfer of the excitation on each period of the modulation is accompanied with transformation of the angular coordinate $\theta$ according to the expanding circle map. (Hereafter we consider the tripling transformations.) As a consequence, the dynamics of the amplitudes in the system will correspond to the presence of the Smale-Williams type attractor in the stroboscopic map.

Let us consider model equations in terms of the complex amplitudes of the following form:

$$
\begin{aligned}
\dot{a}_{1}= & \frac{1}{2}\left[A \cos (2 \pi t / T)-\left|a_{1}\right|^{2}-\left|a_{2}\right|^{2}\right] a_{1}+\frac{1}{2} \varepsilon b_{1}, \\
\dot{a}_{2}=\frac{1}{2}[ & \left.A \cos (2 \pi t / T)-\left|a_{1}\right|^{2}-\left|a_{2}\right|^{2}\right] a_{2}+\frac{1}{2} \varepsilon b_{2}, \\
\dot{b}_{1}= & \frac{1}{2}\left[-A \cos (2 \pi t / T)-\left|b_{1}\right|^{2}-\left|b_{2}\right|^{2}\right] b_{1} \\
& +\frac{1}{2} \varepsilon a_{1}\left(\left|a_{1}\right|^{2}-3\left|a_{2}\right|^{2}\right), \\
\dot{b}_{2}= & \frac{1}{2}\left[-A \cos (2 \pi t / T)-\left|b_{1}\right|^{2}-\left|b_{2}\right|^{2}\right] b_{2} \\
& +\frac{1}{2} \varepsilon a_{2}\left(3\left|a_{1}\right|^{2}-\left|a_{2}\right|^{2}\right) .
\end{aligned}
$$

Here $a_{1,2}$ and $b_{1,2}$ are the complex amplitudes associated with the two subsystems, each composed of a pair of oscillators fed from a separate common energy source. Due to the counterphase modulation of parameters controlling the selfoscillations in the subsystems, they become active alternately; $A$ is the magnitude of the modulation, $T$ is the period of the modulation. The terms proportional to the constant $\varepsilon$ introduce pairwise coupling between the oscillators relating to the two 

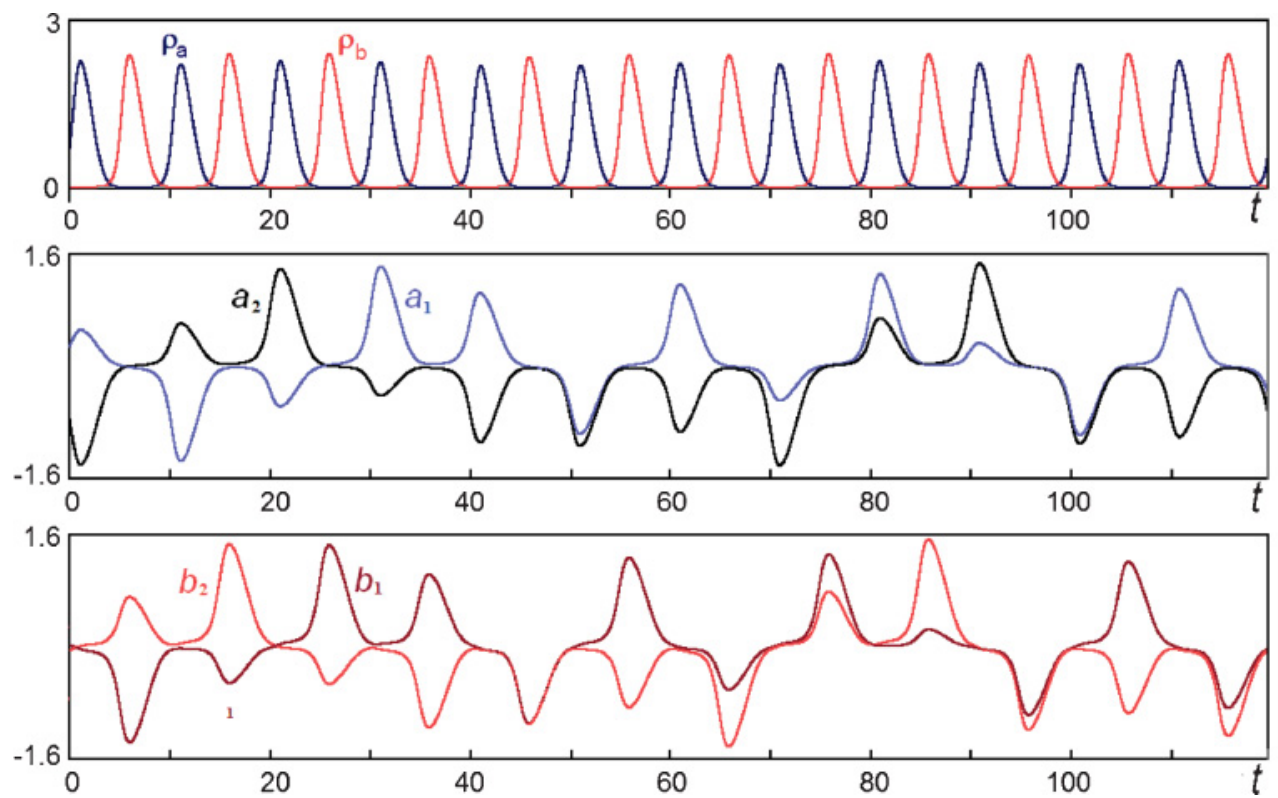

(c)

FIG. 1. (Color online) Typical example of time dependencies for the amplitude variables of four oscillators obtained from numerical solution of Eq. (4) with $T=10, A=3, \varepsilon=0.06$ : (a) pairwise sums of squared amplitudes $\rho_{a}=a_{1}^{2}+a_{2}^{2}$ and $\rho_{b}=b_{1}^{2}+b_{2}^{2}$, and the amplitude variables for (b) the first and (c) the second pair of the oscillators.

subsystems. As explained below, their concrete form is chosen according to the desired mode of operation.

Suppose in a half period of activity of the first pair $(A \cos 2 \pi t / T>0)$ the variables $a_{1,2}$ are characterized by nearly constant amplitudes corresponding to some $\theta: a_{1} \sim$ $\cos \theta, a_{2} \sim \sin \theta$. At the beginning of the activity stage for the second pair $(A \cos 2 \pi t / T<0)$, the excitation of the oscillators is stimulated by the coupling terms in the third and fourth lines of Eq. (2). From the well-known trigonometric relations, one can see that

$$
\begin{aligned}
& \left(\left|a_{1}\right|^{2}-3\left|a_{2}\right|^{2}\right) a_{1} \sim\left(\cos ^{2} \theta-3 \sin ^{2} \theta\right) \cos \theta=\cos 3 \theta, \\
& \left(3\left|a_{1}\right|^{2}-\left|a_{2}\right|^{2}\right) a_{2} \sim\left(3 \cos ^{2} \theta-\sin ^{2} \theta\right) \sin \theta=\sin 3 \theta .
\end{aligned}
$$

Hence, the amplitude ratio for the complex variables $b_{1,2}$ will be determined by the tripled angular variable, $3 \theta$. Next, as the activity stage of the second pair comes to the end, the excitation is transferred back to the first pair. According to the form of the coupling terms in the first and second equations (2), it occurs without change of the $\theta$ variable. So, over the complete period of modulation this variable undergoes the tripling transformation: $\theta_{n+1} \approx 3 \theta_{n}$.

Observe that Eq. (2) is invariant with respect to two phase shifts (i.e., to the variable changes $a_{1}, b_{1} \rightarrow a_{1} e^{i \varphi_{1}}, b_{1} e^{i \varphi_{1}}$ and $a_{2}, b_{2} \rightarrow a_{2} e^{i \varphi_{2}}, b_{2} e^{i \varphi_{2}}$, where $\varphi_{1}$ and $\varphi_{2}$ are arbitrary constants). We can, therefore, restrict ourselves to the description of amplitude dynamics exclusively, using the equations in real variables

$$
\begin{aligned}
& \dot{a}_{1}=\frac{1}{2}\left[A \cos (2 \pi t / T)-a_{1}^{2}-a_{2}^{2}\right] a_{1}+\frac{1}{2} \varepsilon b_{1}, \\
& \dot{a}_{2}=\frac{1}{2}\left[A \cos (2 \pi t / T)-a_{1}^{2}-a_{2}^{2}\right] a_{2}+\frac{1}{2} \varepsilon b_{2}, \\
& \dot{b}_{1}=\frac{1}{2}\left[-A \cos (2 \pi t / T)-b_{1}^{2}-b_{2}^{2}\right] b_{1}+\frac{1}{2} \varepsilon a_{1}\left(a_{1}^{2}-3 a_{2}^{2}\right), \\
& \dot{b}_{2}=\frac{1}{2}\left[-A \cos (2 \pi t / T)-b_{1}^{2}-b_{2}^{2}\right] b_{2}+\frac{1}{2} \varepsilon a_{2}\left(3 a_{1}^{2}-a_{2}^{2}\right) .
\end{aligned}
$$

(Here we allow the variables $a_{1,2}$ and $b_{1,2}$ to be positive or negative, so the true amplitudes of the oscillators correspond to the absolute values of these variables.)

Next, instead of description of the dynamics in terms of continuous time, one can examine the dynamics in discrete time by means of a stroboscopic Poincaré map. In our case, the map $\mathbf{x}_{n+1}=\mathbf{T}\left(\mathbf{x}_{n}\right)$ operates with vectors $\mathbf{x}_{n}=$ $\left\{a_{1}, a_{2}, b_{1}, b_{2}\right\}_{t=n T}$. Although not derived analytically, this map can be obtained easily through computations by solving the differential equations over one period $T$. In the frame of the original equations, the map is eight-dimensional (accounting for the complex nature of the variables $a_{1,2}$ and $b_{1,2}$ ), but for the real-variable equations (4) it is four-dimensional.

In the discussed mode of operation, according to the above argumentation, one can define an angular variable undergoing tripling on successive iterations. Due to compression of the phase volume along other directions in the four-dimensional state space of the Poincaré map of the model in Eq. (4), the attractor of this map is expected to be a variant of the Smale-Williams solenoid arising due to the tripling of a number of coils on each next step of the iterations. It will be a uniformly hyperbolic attractor embedded in the state space of the four-dimensional Poincare map with one-dimensional unstable manifold and three-dimensional stable manifold of every orbit on the attractor.

To illustrate that the model operates in accordance with the above description, let us consider some computational results. Figure 1 shows time dependences for real amplitudes obtained from numerical simulation of equations (4) at appropriately selected parameter values $T=10, A=3, \varepsilon=0.06$. Observe the alternating activity of the first and the second pairs of the oscillators. As shown below, the transformation of the angular variable responsible for distribution of the amplitudes in each pair of oscillators corresponds to the Bernoulli-type map on each modulation period. 

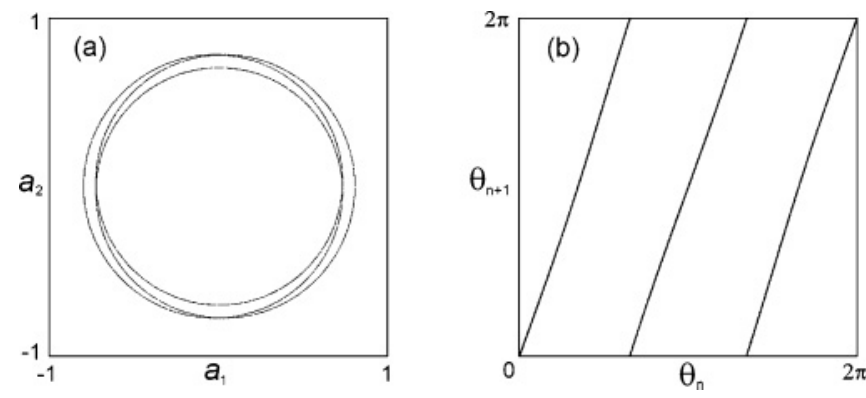

FIG. 2. Attractor in the Poincaré section in two-dimensional projection on the plane of real amplitudes of (a) the first two oscillators and (b) diagram for the angular variables at successive instants $t=n T$ for the system in Eq. (4) with $T=10, \varepsilon=0.06, A=3$.

Figure 2(a) shows the attractor of the Poincare map in projection on a plane of variables relating to one of the alternately exciting subsystems and 2(b) shows a numerically obtained diagram illustrating transformation at successive modulation periods of the angular coordinate responsible for distribution of the amplitudes in each pair of the oscillators constituting the system. The angular variable was evaluated at successive instants $t_{n}=n T$ as $\theta_{n}=\arctan \left[b_{1}(n T) / a_{1}(n T)\right]$, if $a_{1}(n T)>0$, and $\theta_{n}=\arctan \left[b_{1}(n T) / a_{1}(n T)\right]+\pi$ otherwise. The attractor indeed looks like a projection of the SmaleWilliams solenoid containing a fine structure of filaments. It may be seen that for the cyclic coordinate $\theta$ the expanding circle map is recovered with high accuracy producing just the tripling of the variable: One bypass of the circle for preimage implies the three-fold bypass for the image.

Evaluation of the Lyapunov exponents for the model in Eq. (4) is based on simultaneous solution of these equations together with a collection of four linearized variation equations along the reference orbit on the attractor, with Gram-Schmidt orthogonalization and renormalization of the respective perturbation vectors in the course of the procedure (the Benettin algorithm [33]).

Figure 3(a) shows all four Lyapunov exponents for the Poincaré map plotted versus the amplitude of slow modulation $A$ for fixed values of the other parameters $\varepsilon=0.06, T=10$. Panels (b) and (c) represent analogous plots of the Lyapunov exponents versus $\varepsilon$ (at $A=3$ and $T=10$ ) and $T$ (at $A=3$ and $\varepsilon=0.06$ ). Notice that the largest exponent remains almost constant and close to $\ln 3=1.0986 \ldots$ in a wide range of each parameter. This agrees well with the approximation based on the Bernoulli tripling map. Such behavior of the largest Lyapunov exponent responsible for the chaotic nature of the dynamics may be regarded as a confirmation of structural stability of the hyperbolic attractor, which evidently persists in certain domain in the parameter space The other three exponents are all negative and correspond to contraction of the phase volume in directions transversal to the filaments of the Smale-Williams solenoid.

For $A=3, \varepsilon=0.06$ and $T=10$ the Lyapunov exponents for the Poincaré map are

$$
\begin{gathered}
\Lambda_{1}=1.095, \quad \Lambda_{2}=-4.172, \\
\Lambda_{3}=-7.712, \quad \Lambda_{4}=-10.690 .
\end{gathered}
$$

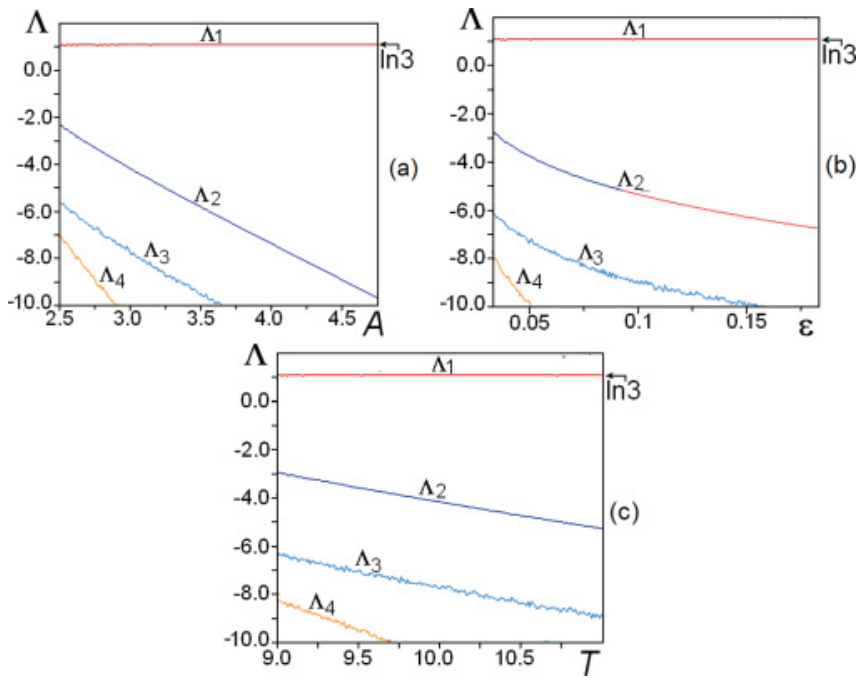

FIG. 3. (Color online) Lyapunov exponents of the Poincaré map for the system in Eq. (4) plotted versus parameters: (a) the dependence on the amplitude of the slow modulation $A$ at $T=10, \varepsilon=0.06$; (b) the dependence on the coupling parameter $\varepsilon$ at $A=3, T=10$; (c) the dependence on the period of the slow modulation $T$ at $A=3$, $\varepsilon=0.06$.

and an estimate of the fractal dimension of the attractor in the Poincaré sections by the Kaplan-Yorke formula yields $D=$ $1+\Lambda_{1} /\left|\Lambda_{2}\right| \approx 1.26$. The noninteger value reflects the fractallike structure of the attractor.

To verify the hyperbolicity of the attractor, we apply the numerical approach (suggested e.g., in Refs. [34-36]). In this procedure, the angles between the directions of growth of small perturbations are calculated forward and backward in time at points of a reference trajectory. Absence of angles close to zero indicates that the dynamics is hyperbolic. If the angle distribution demonstrates nonzero probability of very small angles, it suggests the presence of tangencies between stable and unstable manifolds and thus implies nonhyperbolicity.

In our case for the Poincare map of the real-amplitude equations (4), the unstable manifold is one-dimensional, and the stable manifold is three-dimensional. An appropriate modification of the above method was described in Refs. [24,25,37]. First, we generate a representative orbit on the attractor by computing Eqs. (4) over a sufficiently long time interval. Next, we compute variation equations for perturbations of the orbit forward in time, normalizing the vector $\mathbf{a}(t)=$ $\left\{\tilde{a}_{1}(t), \tilde{a}_{2}(t), \tilde{b}_{1}(t), \tilde{b}_{2}(t)\right\}$ at each integration step to preclude divergence. Then, we compute three replicas of the variation equations with randomly chosen initial conditions backward in time to find three vectors $\mathbf{b}(t), \mathbf{c}(t)$, and $\mathbf{d}(t)$, performing Gram-Schmidt reorthonormalization of the vectors at each integration step to avoid divergence and predominance of one of the vectors.

At each stroboscopic section $t_{n}=n T$, the vector $\mathbf{a}_{n}=\mathbf{a}\left(t_{n}\right)$ defines tangent direction to the unstable manifold and the span of $\left\{\mathbf{b}_{n}, \mathbf{c}_{n}, \mathbf{d}_{n}\right\}=\left\{\mathbf{b}\left(t_{n}\right), \mathbf{c}\left(t_{n}\right), \mathbf{d}\left(t_{n}\right)\right\}$ corresponds to the tangent subspace to the three-dimensional stable manifold. To evaluate the angle $\alpha$ between the unstable and stable manifolds, we determine a vector $\mathbf{v}_{n}$ transversal to the stable manifold by solving the set of algebraic linear equations 


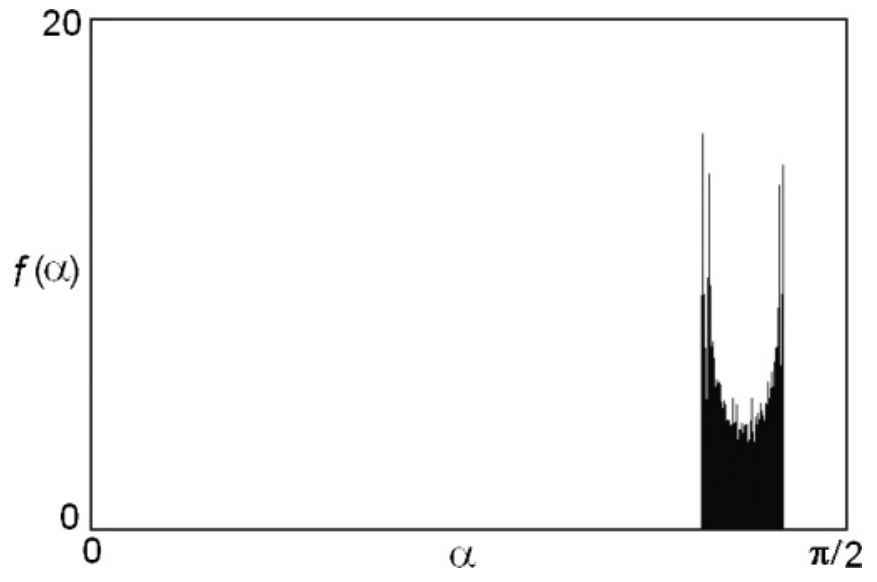

FIG. 4. An estimate of the density function for the distribution of angles between the local stable and unstable manifolds for the attractor in the stroboscopic Poincaré map obtained numerically at $T=10, A=3, \varepsilon=0.06$ for the model system in Eq. (4) represented in real amplitudes. Observe the clearly expressed separation of the distribution from zero angles that is the indicator of the hyperbolicity.

$\mathbf{v}_{n} \cdot \mathbf{b}_{n}=0, \mathbf{v}_{n} \cdot \mathbf{c}_{n}=0, \mathbf{v}_{n} \cdot \mathbf{d}_{n}=0$. Then, we calculate the angle $\beta_{n} \in[0, \pi / 2]$ from $\cos \beta_{n}=\left|\mathbf{v}_{n} \cdot \mathbf{a}_{n}\right| /\left|\mathbf{v}_{n}\right|\left|\mathbf{a}_{n}\right|$, and set $\alpha_{n}=\beta_{n}-\pi / 2$. Figure 4 shows the density function estimated from the histogram of the computed array of $\alpha_{n}$. Since the distribution is clearly separated from zero angles, the test confirms the hyperbolicity of the attractor.

For the original set of equations (2) in complex amplitudes evaluation of the Lyapunov exponents in computations yields

$$
\begin{gathered}
\Lambda_{1}=1.097, \quad \Lambda_{2}=0.000 \\
\Lambda_{3}=0.000, \quad \Lambda_{4}=-4.166 \\
\Lambda_{5}=-5.366, \quad \Lambda_{6}=-5.365 \\
\Lambda_{7}=-7.556, \quad \Lambda_{8}=-10.637 .
\end{gathered}
$$

The largest exponent is of the same value as in the realamplitude equations up to numerical errors and it is close to $\ln 3$. The second and the third exponents are zero (up to inaccuracy of the computations); they are evidently associated with the neutral perturbations of the above-mentioned two phase shifts leaving the equations invariant. All other exponents are negative; among them $\Lambda_{7}$ and $\Lambda_{8}$ coincide with certain negative exponents from the spectrum of the four-dimensional model, while $\Lambda_{5}$ and $\Lambda_{6}$ are special for the complex-amplitude version of the model.

Estimate of the fractal dimension of the attractor in the Poincaré sections by the Kaplan-Yorke formula yields $D=$ $3+\left(\Lambda_{1}+\Lambda_{2}+\Lambda_{3}\right) /\left|\Lambda_{4}\right| \approx 3.26$. This attractor embedded in the eight-dimensional state space of the Poincare map has to be regarded as relating to the class of partially hyperbolic attractors because of presence of center subspace associated with the phase variables $\varphi_{1}$ and $\varphi_{2}$ and the corresponding zero-valued two Lyapunov exponents. In the model under consideration this remark is not so essential: The invariance of the equations (2) in respect to the phase shifts is exact; it means that one can accept a rightful agreement not to distinguish states distinct only in the phases, and, in this sense, treat the dynamics as true uniformly hyperbolic. However, in systems for which description in terms of slow complex amplitudes is approximate (like those discussed in the next two subsections), one may expect peculiarities associated with some features of the partially hyperbolic attractor. If the deflections from the slow-amplitude approximation are small enough, it may be thought that the dynamics of the amplitude variables will retain its character because of the intrinsic roughness of the hyperbolic attractor.

\section{MODEL OF TWO ALTERNATELY EXCITED PAIRS OF VAN DER POL OSCILLATORS WITH EQUAL NATURAL FREQUENCIES}

Let us now turn our attention toward model systems composed of van der Pol oscillators. The van der Pol oscillator is a well known, popular, and significant paradigm model of a self-oscillatory system. It is often applied in the context of electrical, mechanical, chemical, and biological oscillations; and the same may be said in relation to composite schemes based on such elements.

In this section we assume that all partial oscillators used to build a composite system have equal natural frequency $\omega_{0}$, and consider the following set of equations

$$
\begin{gathered}
\ddot{x}_{1}-\left[A \cos (2 \pi t / T)-x_{1}^{2}-x_{2}^{2}\right] \dot{x}_{1}+\omega_{0}^{2} x_{1}=\varepsilon \dot{y}_{1}, \\
\ddot{x}_{2}-\left[A \cos (2 \pi t / T)-x_{1}^{2}-x_{2}^{2}\right] \dot{x}_{2}+\omega_{0}^{2} x_{2}=\varepsilon \dot{y}_{2}, \\
\ddot{y}_{1}-\left[-A \cos (2 \pi t / T)-y_{1}^{2}-y_{2}^{2}\right] \dot{y}_{1}+\omega_{0}^{2} y_{1}=\varepsilon\left(x_{1}^{2}-3 x_{2}^{2}\right) \dot{x}_{1}, \\
\ddot{y}_{2}-\left[-A \cos (2 \pi t / T)-y_{1}^{2}-y_{2}^{2}\right] \dot{y}_{2}+\omega_{0}^{2} y_{2}=\varepsilon\left(3 x_{1}^{2}-x_{2}^{2}\right) \dot{x}_{2} .
\end{gathered}
$$

Here we have two pairs of the self-oscillators. Each pair, characterized by the generalized coordinate variables $x_{1,2}$ and $y_{1,2}$, respectively, is supposed to get supply from the common source, as seen from the structure of the expressions in square brackets. The two pairs become active in turn because of the periodic modulation of the parameters responsible for the Andronov-Hopf bifurcation in the van der Pol oscillators; the intensity of the modulation is characterized by parameter $A$, and its period by the constant $T$. The coupling terms proportional to the constant $\varepsilon$ ensure transfer of the excitation between the first and second oscillators of each pair; their structure is similar to that postulated in the previous section. Note that the transfer of the excitation occurs under a resonance condition through the first harmonic component of the righthand terms in the equations.

As shown in Appendix, using the approximation of the slow-amplitude approach $[2,22,41,42]$, the system is reduced just to the form, which corresponds to the real amplitude equations (4). Hence, its operation must be similar to that considered in the previous section. In view of the structural stability of the hyperbolic attractor of the model in Eq. (4), the approximate correspondence is expected to take place, at least for parameters in the range of applicability of the description in terms of the amplitude equations.

Figure 5 shows wave forms generated by the system as obtained from numerical solution of Eqs. (7) for $A=3$, $T=10, \varepsilon=0.06, \omega_{0}=2 \pi$. Panels (a) and (b) represent the plots for the pairwise coupled oscillators. Observe that the first and second subsystems are active alternately, and that the distribution of the intensity of the oscillations varies 

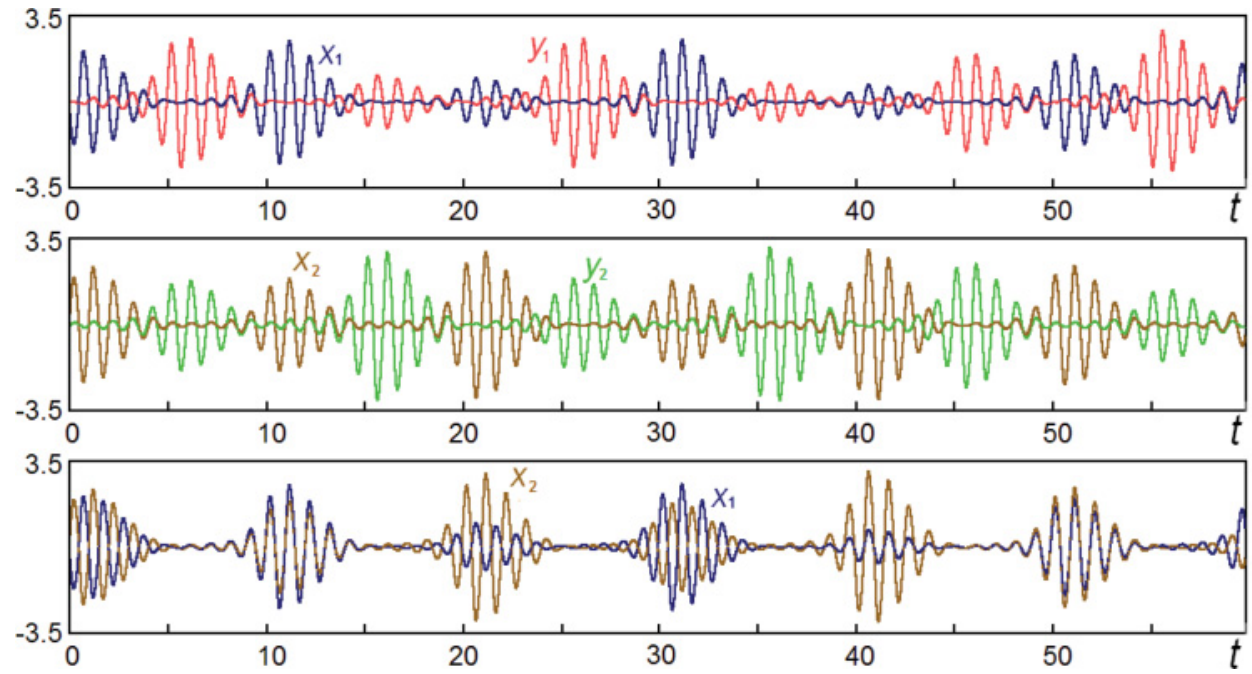

FIG. 5. (Color online) Wave forms generated by the system in Eq. (7) for $A=3, T=10, \varepsilon=0.06, \omega_{0}=2 \pi$.

drastically between successive stages of activity. Panel (c) shows the wave forms of two oscillators relating to one and the same subsystem; observe that the phases of both oscillators are the same, at least as distinguishable from the plot. (Actually, this phase varies slowly on large time scales and represents a special variable not involved in the hyperbolic structure; due to this circumstance the attractor in the stroboscopic map of this system relates, as believed, to the partially hyperbolic class.)

It is again convenient to introduce a description in terms of a stroboscopic Poincaré map. Now, the map $\mathbf{x}_{n+1}=\mathbf{T}\left(\mathbf{x}_{n}\right)$ operates with vectors $\mathbf{x}_{n}=\left\{x_{1}, u_{1}, x_{2}, u_{2}, y_{1}, v_{1}, y_{2}, v_{2}\right\}_{t=n T}$, where $u_{1,2}=\dot{x}_{1,2} / \omega_{0}$ and $v_{1,2}=\dot{y}_{1,2} / \omega_{0}$ are normalized generalized velocities for the respective oscillators. The Poincaré map can be obtained computationally by solving the differential equations (7) for one period $T$.

To proceed, we need to introduce an angular variable characterizing the distribution of amplitudes for the oscillators constituting one subsystem, expressed via the dynamical variables $\left(x_{1,2}, u_{1,2}\right)$. In the context of the present model this is a more subtle matter than that in the previous section. (A straightforward definition like $\arctan \left[\sqrt{x_{2}^{2}+u_{2}^{2}} / \sqrt{x_{1}^{2}+u_{1}^{2}}\right]$ is not satisfactory as it deals with only one of four quadrants for the angular variable; to illustrate the relation to the Smale-Williams solenoid the angular variable needs to be allowed to take values corresponding to the entire circle.) The following approach is found to be successful. First, select a pair from $\left(x_{1}, u_{1}\right)$ and $\left(x_{2}, u_{2}\right)$ with larger $r_{i}^{2}=x_{i}^{2}+u_{i}^{2}$. Then, if $\left|x_{i}\right|>\left|u_{i}\right|$, we set $s=\operatorname{sgn}\left(x_{i}\right)$, otherwise, $s=\operatorname{sgn}\left(u_{i}\right)$, and $\xi=s x_{i} / r_{i}, \eta=s u_{i} / r_{i}$. Now, before and after each step of the Poincaré map, evaluate $X=\xi x_{1}+\eta u_{1}, Y=\xi x_{2}+\eta u_{2}$ and $X^{\prime}=\xi x_{1}^{\prime}+\eta u_{1}^{\prime}, Y^{\prime}=\xi x_{2}^{\prime}+\eta u_{2}^{\prime}$, where the prime marks the updated variables. (The coefficients $\xi$ and $\eta$ are accepted to be identical in the course of evaluation both of $X, Y$ and $X^{\prime}, Y^{\prime}$.) Finally, set $\theta=\arg (X+i Y)$ and $\theta^{\prime}=\arg \left(X^{\prime}+i Y^{\prime}\right)$ to plot along the axes on the diagram for the angular coordinate.

Figure 6 shows the attractor in the Poincare section on the plane of variables $X, Y$ [panel (a)] and the iteration diagram for the angular variable $\theta$ [panel (b)]. In comparison with Fig. 2 the attractor appears as a formation widened in direction transversal to the coils. It is, as mentioned above, because a system now has a slowly evolving phase variable. Nevertheless, some fine structure inherited from the Cantor-like arrangement of the solenoid is distinguishable on the plot. As to the diagram for the angular variable, due to its proper definition, the plot looks very similar to that of Fig. 2(b) associated with the true uniformly hyperbolic attractor. Hence, one may assert that the hyperbolic component actually exists and that its intrinsic structural stability is conserved in spite of the perturbations arising from the dynamics of the phase variable.

Figure 7 shows results of a computation of the spectrum of Lyapunov exponents for the Poincare map of the system in Eq. (7). In panel (a) they are plotted versus the modulation intensity at $T=10, \varepsilon=0.06, \omega_{0}=2 \pi$. Panels (b) and (c) are analogous plots for the Lyapunov exponents versus the coupling parameter $\varepsilon$ (at $A=3, T=10, \omega_{0}=2 \pi$ ) and versus the modulation period $T$ (at $A=3, \varepsilon=0.06, \omega_{0}=2 \pi$ ). Observe that the largest exponent remains close to $\ln 3$ in a wide range. This confirms the robust nature of the motion associated with the hyperbolic component. The second exponent is indistinguishable from zero in the computations, and is obviously associated with the slow-evolving phase variable. Other exponents are all negative. Particularly, at
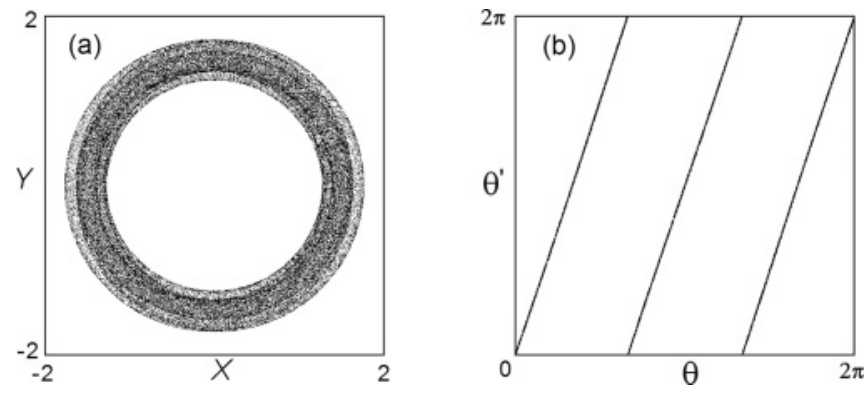

FIG. 6. (a) Attractor in the Poincaré section in two-dimensional projection on the plane of the auxiliary variables introduced in the text and characterizing amplitudes of the first two oscillators and (b) diagram for the angular variables at successive instants $t=n T$ for the system in Eq. (7) at $A=3, T=10, \varepsilon=0.06, \omega_{0}=2 \pi$. 


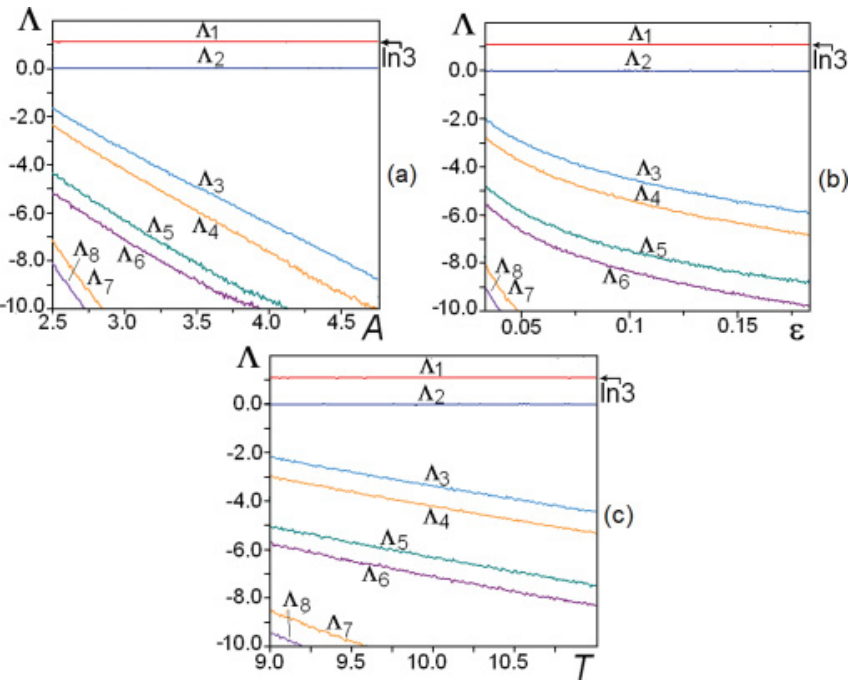

FIG. 7. (Color online) Lyapunov exponents of the Poincaré map for the system in Eq. (7) plotted versus parameters: (a) the dependence on the amplitude of the slow modulation $A$ at $T=10, \varepsilon=0.06$, $\omega_{0}=2 \pi$; (b) the dependence on the coupling parameter $\varepsilon$ at $A=3$, $T=10, \omega_{0}=2 \pi ;$ (c) the dependence on the period of the slow modulation $T$ at $A=3, \varepsilon=0.06, \omega_{0}=2 \pi$.

$A=3, T=10, \varepsilon=0.06, \omega_{0}=2 \pi$ we obtain

$$
\begin{array}{cc}
\Lambda_{1}=1.099, & \Lambda_{2}=0.000, \\
\Lambda_{3}=-3.373, & \Lambda_{4}=-4.198, \\
\Lambda_{5}=-6.326, & \Lambda_{6}=-7.097, \\
\Lambda_{7}=-11.041, & \Lambda_{8}=-12.020 .
\end{array}
$$

It is interesting to compare these values with the results for the uniformly hyperbolic attractor of the four-dimensional realamplitude system. (We recall that the Eqs. (7) reduce to that system in the approximation of the slow-varying amplitudes, see Appendix.) As one can see the values of $\Lambda_{1}, \Lambda_{4}, \Lambda_{6}, \Lambda_{7}$ from the list in Eq. (8) agree well with the values of $\Lambda_{1}, \Lambda_{2}$, $\Lambda_{3}, \Lambda_{4}$ from the list in Eq. (5). The dimension of the attractor in the Poincare map in the present case is estimated from the Kaplan-Yorke formula as $D=2+\left(\Lambda_{1}+\Lambda_{2}\right) /\left|\Lambda_{3}\right| \approx 2.33$.

\section{MODEL OF TWO ALTERNATELY EXCITED PAIRS OF NONRESONANT VAN DER POL OSCILLATORS}

Let us examine another version of a system composed of van der Pol oscillators, which corresponds more closely to the original model in Eq. (2), and is governed by the set of equations

$$
\begin{gathered}
\ddot{x}_{1}-\left[A \cos (2 \pi t / T)-x_{1}^{2}-\frac{1}{2} x_{2}^{2}\right] \dot{x}_{1}+\omega_{1}^{2} x_{1}=\varepsilon \dot{y}_{1}, \\
\ddot{x}_{2}-\left[A \cos (2 \pi t / T)-\frac{1}{2} x_{1}^{2}-x_{2}^{2}\right] \dot{x}_{2}+\omega_{2}^{2} x_{2}=\varepsilon \dot{y}_{2}, \\
\ddot{y}_{1}-\left[-A \cos (2 \pi t / T)-y_{1}^{2}-\frac{1}{2} y_{2}^{2}\right] \dot{y}_{1}+\omega_{1}^{2} y_{1}=\varepsilon\left(x_{1}^{2}-\frac{3}{2} x_{2}^{2}\right) \dot{x}_{1}, \\
\ddot{y}_{2}-\left[-A \cos (2 \pi t / T)-\frac{1}{2} y_{1}^{2}-y_{2}^{2}\right] \dot{y}_{2}+\omega_{2}^{2} y_{2}=\varepsilon\left(\frac{3}{2} x_{1}^{2}-x_{2}^{2}\right) \dot{x}_{2} .
\end{gathered}
$$

Unlike the model in Eq. (7), the first and second oscillator of the alternately excited subsystems are now characterized by different natural frequencies $\omega_{1}$ and $\omega_{2}$, which are assumed not to satisfy low-order resonant conditions. Next, the factors $1 / 2$ are introduced at some terms to ensure correspondence with the system in Eq. (2) in the slow complex amplitude approach for the nonresonant situation (see Appendix). Nevertheless, the transfer of the excitation between the first and second oscillators of each subsystem due to the coupling terms in the right-hand parts remains resonant as the natural frequencies coincide for the oscillators marked with the same subscript 1 or 2 in both subsystems. Operation of the system is similar to that considered in the context of the previous models. In an approximate description based on the real amplitude equations (4), we have the hyperbolic attractor of Smale-Williams type in the stroboscopic map. Because of its structural stability, it is natural to expect that the robust chaotic dynamics of the amplitudes survive in the system (9) as well, although the attractor of the complete set of equations will relate to the partially hyperbolic class.

To illustrate the dynamics we select the parameters $A=3$, $T=10, \varepsilon=0.06$, like in the previous sections. With respect to the natural frequencies $\omega_{1}$ and $\omega_{2}$, it is appropriate to choose them in a ratio given by two successive Fibonacci numbers to avoid at least low order resonances; concretely, we set $\omega_{1}=$ $5 \omega_{0}, \omega_{2}=8 \omega_{0}$ with $\omega_{0}=2 \pi$.

Figure 8 shows wave forms generated by the system as obtained from numerical solution of Eqs. (9). Panels (a) and (b) represent the plots for the generalized coordinates of the pairwise coupled oscillators. The first and second subsystems are active alternately, and the intensities of the oscillations vary on successive stages in a randomlike fashion.

Like in the previous section, we must pay special attention to definition of an appropriate angular variable to illustrate the link with the Smale-Williams attractor in the amplitude dynamics. Now, the definition must be designed to disregard two phase variables relating to the oscillators marked with
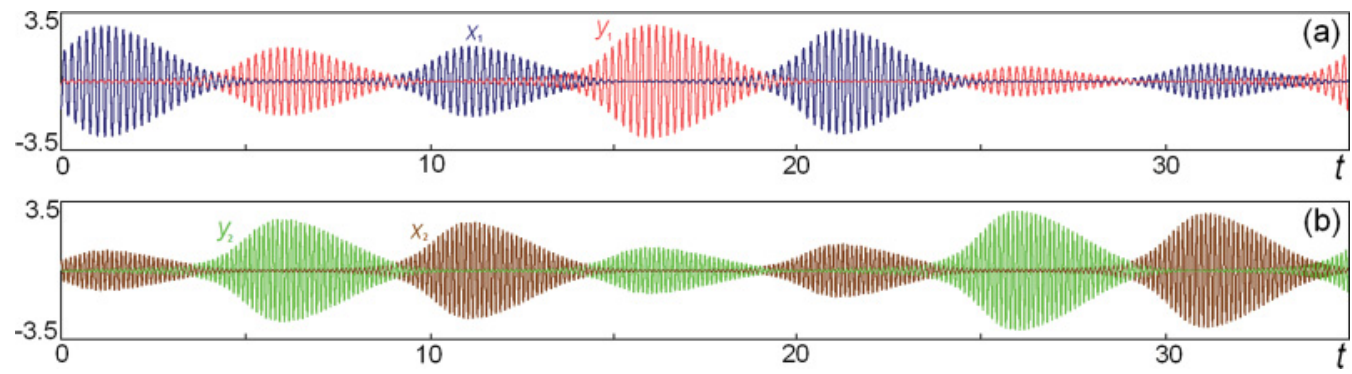

FIG. 8. (Color online) Typical pattern of time dependencies for coordinate variables of four oscillators obtained from numerical solution of Eqs. (9) at $T=10, A=3, \varepsilon=0.06, \omega_{1}=10 \pi, \omega_{2}=16 \pi$. 

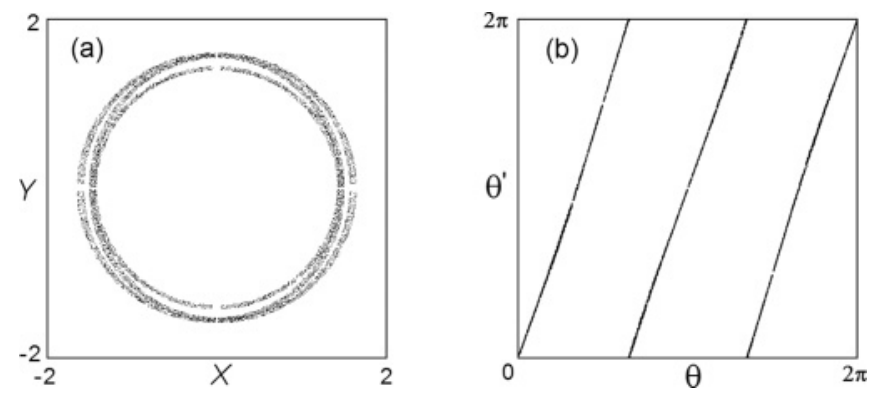

FIG. 9. (a) Attractor in the Poincaré section in two-dimensional projection on the plane of two real variables relating to the first two oscillators of the system in Eq. (9) (see text). (b) Diagram for the angular variables for the system in Eq. (9). Parameters are $T=10$, $\varepsilon=0.06, A=3, \omega_{1}=10 \pi, \omega_{2}=16 \pi$.

subscripts 1 and 2, respectively. The following evaluation routine is appropriate. For $i=1,2$, if $\left|x_{i}\right|>\left|u_{i}\right|$, set $s_{i}=\operatorname{sgn}\left(x_{i}\right)$, otherwise, $s_{i}=\operatorname{sgn}\left(u_{i}\right)$. Then, before and after each step of the Poincaré map, evaluate $X=s_{1} \sqrt{x_{1}^{2}+u_{1}^{2}}, Y=s_{2} \sqrt{x_{2}^{2}+u_{2}^{2}}$ and $X^{\prime}=s_{1} \sqrt{x_{1}^{\prime 2}+u_{1}^{\prime 2}}, Y^{\prime}=s_{2} \sqrt{{x_{2}^{\prime}}^{2}+u_{2}^{\prime 2}}$, where the prime marks the updated variables. (The factors $s_{1,2}$ are identical in evaluation both of $X, Y$ and $X^{\prime}, Y^{\prime}$.) Finally, set $\theta=$ $\arg (X+i Y)$ and $\theta^{\prime}=\arg \left(X^{\prime}+i Y^{\prime}\right)$.

Figure 9 shows the attractor in the Poincaré section on the plane of variables $X, Y$ [panel (a)] and the iteration diagram for the angular variable $\theta$ [panel (b)]. The attractor looks similar to that shown in Fig. 2 with a certain transversal widening occurring apparently because of the slow evolution of the phase variables. The diagram for the angular variable looks very similar to that of Fig. 2(b) associated with the uniformly hyperbolic Smale-Williams attractor.

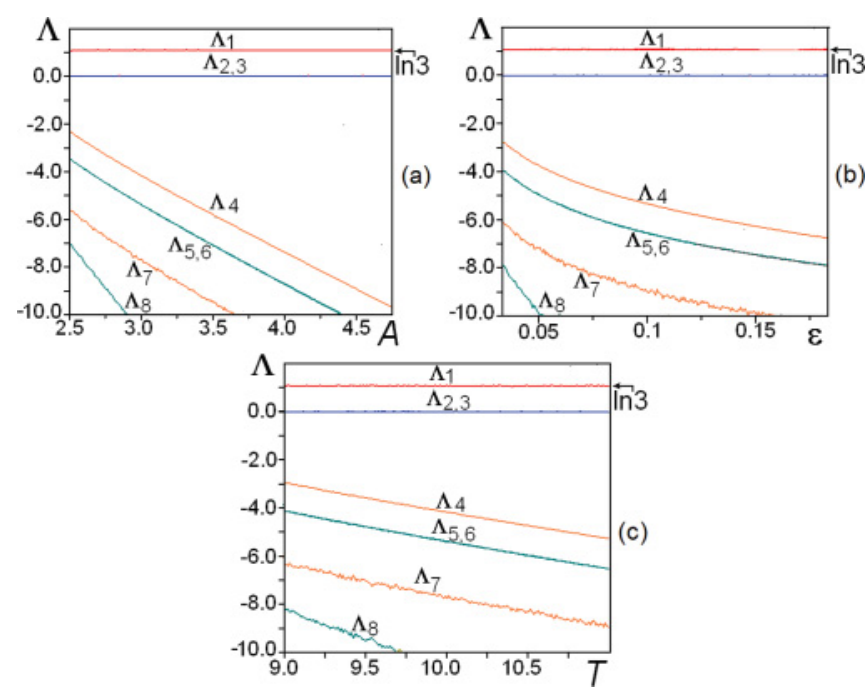

FIG. 10. (Color online) Lyapunov exponents of the Poincaré map for the system in Eq. (9) plotted versus parameters: (a) the dependence on the amplitude of the slow modulation $A$ at $T=10, \varepsilon=0.06$, $\varepsilon=0.06$; (b) the dependence on the coupling parameter $\varepsilon$ at $A=3$, $T=10$; (c) the dependence on the period of the slow modulation $T$ at $A=3, \varepsilon=0.06$. The values of the other parameters are $\omega_{1}=10 \pi$, $\omega_{2}=16 \pi$.
Figure 10 shows Lyapunov exponents for the Poincaré map of the system in Eq. (9) versus parameter $A$ [panel (a)], versus parameter $\varepsilon$ [panel (b)], and versus parameter $T$ [panel (c)] at fixed values of the other parameters. Again, the largest exponent is close to $\ln 3$ in a wide parameter range. The second and third exponents are nearly zero being associated obviously with two slow-evolving phase variables. Other exponents are all negative. Particularly, for $A=3, T=10, \varepsilon=0.06$ we have

$$
\begin{gathered}
\Lambda_{1}=1.081, \quad \Lambda_{2}=0.000, \\
\Lambda_{3}=0.000, \quad \Lambda_{4}=-4.173, \\
\Lambda_{5}=-5.385, \quad \Lambda_{6}=-5.386, \\
\Lambda_{7}=-7.717, \quad \Lambda_{8}=-10.632 .
\end{gathered}
$$

These results are remarkable close to the data in Eq. (6) corresponding to the description of the system in terms of slow complex amplitudes. The Kaplan-Yorke estimate of the dimension is $D \approx 3.26$.

\section{CONCLUSION}

In this article we have advanced an approach to the design of systems with attractor of Smale-Williams type in the stroboscopic map for the amplitudes of oscillators constituting the system. The approach is based on the manipulation by the angular variable describing distribution of amplitudes for self-oscillators, which are fed from a common supply of energy. By this manipulation the angular variable undergoes transformation according to expanding circle map on each time period of the externally forced periodic parameter modulation. The proposed principle can be implemented, for example, in systems of electronics and nonlinear optics for the generation of robust chaos. Moreover, on this principle, one can build many models manifesting various interesting phenomena of complex dynamics, like it was done with systems based on manipulation by phases of successively generated oscillatory trains (e.g., Arnold cat map dynamics [26,38], complex analytic dynamics with Mandelbrot and Julia sets [39], robust strange nonchaotic attractor [40], etc.).

A subtle point in the present study is that the feasible examples composed of van der Pol oscillators and discussed in Secs. III and IV are reduced to the equation with a uniformly hyperbolic attractor only in a certain approximation corresponding to the description in terms of real amplitudes while neglecting the phases. When the phases are taken into account, the attractor in principle has to be regarded as belonging to the class of partially hyperbolic attractors. Because of the structural stability for the attractor of the amplitude equations, it is natural to suppose that the dynamics of the amplitudes is not affected by influence of the dynamics of the phases, and therefore this dynamics preserves the properties intrinsic to the uniformly hyperbolic attractor (one can talk of the hyperbolic component of the motion). Data of numerical simulations seem to confirm this assumption.

Quantitatively, features of the partial hyperbolicity reveal themselves, for example, in Lyapunov spectra (presence of zero or close to zero exponents) and in dimensional characteristics. Say the integer part of the fractal dimension 
for the Poincaré map attractor equals the dimension of the center subspace plus one (the dimension of the unstable subspace), and the fractional part of the dimension estimated from the Kaplan-Yorke formula may depend on the terms of the Lyapunov spectrum outside those relating to the amplitude dynamics (see the final paragraph of Sec. III). Surely, mathematical justification of these observations would be desirable.

In this frame, it is worth noting that elaboration of the mathematically sound criteria for partial hyperbolicity, which would allow verification through computations (like the cone criterion, or analysis of the distribution of angles of intersection of stable and unstable subspaces for the uniform hyperbolicity) surely deserves attention as well as the question to what extent physically meaningful roughness of chaotic dynamics may be associated with the partially hyperbolic attractors.

In any case, the material presented in this article is of practical interest in a frame of design of robust chaos generators (e.g., for communication systems, cryptography, and random number generation). At the same time it is of theoretical interest by providing deep and well-developed concepts of the mathematical hyperbolic theory with a physical content.

\section{ACKNOWLEDGMENTS}

O.B.I. acknowledges partial support from the President of Russian Federation, Grant No. MK-905.2010.2. S.P.K. acknowledges partial support from the Russian Foundation for Basic Research, Grant No. 09-02-00426.

\section{APPENDIX: SLOW-AMPLITUDE DESCRIPTION OF MODELS COMPOSED OF VAN DER POL OSCILLATORS}

Let us apply the method of slow complex amplitudes [2,22,41,42] first to the system in Eq. (9). With this purpose, we set

$$
\begin{aligned}
& x_{1}=a_{1} e^{i \omega_{1} t}+a_{1}^{*} e^{-i \omega_{1} t}, \\
& x_{2}=a_{2} e^{i \omega_{2} t}+a_{2}^{*} e^{-i \omega_{2} t}, \\
& y_{1}=b_{1} e^{i \omega_{1} t}+b_{1}^{*} e^{-i \omega_{1} t}, \\
& y_{2}=b_{2} e^{i \omega_{2} t}+b_{2}^{*} e^{-i \omega_{2} t},
\end{aligned}
$$

and

$$
\begin{aligned}
& \dot{x}_{1}=i \omega_{1} a_{1} e^{i \omega_{1} t}-i \omega_{1} a_{1}^{*} e^{-i \omega_{1} t} \\
& \dot{x}_{2}=i \omega_{2} a_{2} e^{i \omega_{2} t}-i \omega_{2} a_{2}^{*} e^{-i \omega_{2} t} \\
& \dot{y}_{1}=i \omega_{1} b_{1} e^{i \omega_{1} t}-i \omega_{1} b_{1}^{*} e^{-i \omega_{1} t} \\
& \dot{y}_{2}=i \omega_{2} b_{2} e^{i \omega_{2} t}-i \omega_{2} b_{2}^{*} e^{-i \omega_{2} t}
\end{aligned}
$$

where complex amplitudes $a_{1,2}(t), b_{1,2}(t)$ are assumed to be functions of time. Simultaneous validity of Eqs. (A1) and (A2) implies that the following relations must take place

$$
\begin{aligned}
& \dot{a}_{1} e^{i \omega_{1} t}+\dot{a}_{1}^{*} e^{-i \omega_{1} t}=0, \\
& \dot{a}_{2} e^{i \omega_{2} t}+\dot{a}_{2}^{*} e^{-i \omega_{2} t}=0, \\
& \dot{b}_{1} e^{i \omega_{1} t}+\dot{b}_{1}^{*} e^{-i \omega_{1} t}=0, \\
& \dot{b}_{2} e^{i \omega_{2} t}+\dot{b}_{2}^{*} e^{-i \omega_{2} t}=0 .
\end{aligned}
$$

(Acceptance of these relations is rightful: As we introduce four complex variables instead of four real ones, we are free to impose these additional conditions.)

Now, we substitute the variables $x$ and $y$ expressed in terms of the complex amplitudes in Eqs. (9) and, accounting for the relations in Eq. (A3), replace the derivatives of the conjugate variables through the complex amplitudes themselves (e.g. $\dot{a}_{1}^{*} e^{-i \omega_{1} t}=-\dot{a}_{1} e^{i \omega_{1} t}$ etc.). The resulting set of equations is exact, but a cumbersome representation of the original equations in the new variables $a_{1}, a_{2}, b_{1}, b_{2}$. The next step is account for the slow-varying nature of the complex amplitudes. We multiply the equations for variables $a_{1}$ and $b_{1}$ by $e^{-i \omega_{1} t}$, and the equations for $a_{2}$ and $b_{2}$ by $e^{-i \omega_{2} t}$ and perform the averaging over the fast oscillations. In the absence of loworder resonances (relations of type $p \omega_{1}=q \omega_{2}$ with integer coefficients less than 4 ), this corresponds simply to removing all terms in the equations containing the exponentials. After some obvious, though cumbersome algebraic manipulations, we obtain exactly the Eqs. (2) for the complex amplitudes $a_{1}$, $a_{2}, b_{1}, b_{2}$.

Let us finally consider the application of this method to Eqs. (7). Now, the natural frequencies of all oscillators are equal, and we use the relations in Eqs. (A1)-(A3) setting $\omega_{1,2}=\omega_{0}$. After substitution into Eqs. (7) one observes that some additional terms survive in the averaged relations because of the equality of the frequencies, and the resulting set of equations reads

$$
\begin{aligned}
\dot{a}_{1}= & \frac{1}{2}\left[A \cos (2 \pi t / T) a_{1}-\left|a_{1}\right|^{2} a_{1}-2\left|a_{2}\right|^{2} a_{1}+a_{1}^{*} a_{2}^{2}\right]+\frac{1}{2} \varepsilon b_{1}, \\
\dot{a}_{2}= & \frac{1}{2}\left[A \cos (2 \pi t / T) a_{2}-2\left|a_{1}\right|^{2} a_{2}+a_{1}^{2} a_{2}^{*}-\left|a_{2}\right|^{2} a_{2}\right]+\frac{1}{2} \varepsilon b_{2}, \\
\dot{b}_{1}= & \frac{1}{2}\left[-A \cos (2 \pi t / T) b_{1}-\left|b_{1}\right|^{2} b_{1}-2\left|b_{2}\right|^{2} b_{1}+b_{1}^{*} b_{2}^{2}\right] \\
& +\frac{1}{2} \varepsilon\left(\left|a_{1}\right|^{2} a_{1}-6\left|a_{2}\right|^{2} a_{1}+3 a_{1}^{*} a_{2}^{2}\right), \\
\dot{b}_{2}= & \frac{1}{2}\left[-A \cos (2 \pi t / T) b_{2}-2\left|b_{1}\right|^{2} b_{2}+b_{1}^{2} b_{2}^{*}-\left|b_{2}\right|^{2} b_{2}\right] \\
& +\frac{1}{2} \varepsilon\left(6\left|a_{1}\right|^{2} a_{2}-3 a_{1}^{2} a_{2}^{*}-\left|a_{2}\right|^{2} a_{2}\right) .
\end{aligned}
$$

Note that these equations differ from the initial model in Eq. (2). However, their structure allows us to consider a situation when all the amplitudes are real. With this additional assumption, the Eqs. (A4) reduce precisely to the realamplitude equations (4).

Lyapunov exponents computed for the attractor of the stroboscopic Poincaré map for the system in Eq. (A4) with $A=3, T=10, \varepsilon=0.06$

$$
\begin{array}{cl}
\Lambda_{1}=1.097, & \Lambda_{2}=0.000 \\
\Lambda_{3}=-3.414, & \Lambda_{4}=-4.167 \\
\Lambda_{5}=-6.204, & \Lambda_{6}=-7.584 \\
\Lambda_{7}=-10.834, & \Lambda_{8}=-11.845
\end{array}
$$

are found to be in remarkable agreement with the results in Eq. (8). This confirms the applicability of the slow-amplitude method in the operation modes we deal with. 
[1] L. Glass and M. C. Mackey, From Clocks to Chaos: The Rhythms of Life (Princeton University Press, Princeton, 1988).

[2] M. I. Rabinovich and D. I. Trubetskov, Oscillations and Waves in Linear and Nonlinear Systems (Kluwer Academic, Dordrecht, 1989).

[3] S. K. Scott, Chemical Chaos (Oxford University Press, Oxford, 1993).

[4] E. Mosekilde, Topics in Nonlinear Dynamics: Applications to Physics, Biology, and Economic Systems (World Scientific, Singapore, 1998).

[5] V. S. Anishchenko, Nonlinear Dynamics of Chaotic and Stochastic Systems: Tutorial and Modern Developments (Springer, Berlin, 2002).

[6] J. W. Haefner, Modeling Biological Systems: Principles and Applications (Springer, Berlin, 2005).

[7] A. Loskutov, Phys. Usp. 53, 1257 (2010).

[8] S. Smale, Bull. Amer. Math. Soc. (NS) 73, 747 (1967).

[9] L. Shilnikov, Int. J. Bifurc. Chaos Appl. Sci. Engng. 7, 1953 (1997).

[10] D. V. Anosov, G. G. Gould, S. K. Aranson, V. Z. Grines, R. V. Plykin, A. V. Safonov, E. A. Sataev, S. V. Shlyachkov, V. V. Solodov, A. N. Starkov, and A. M. Stepin, in Encyclopaedia of Mathematical Sciences, Vol. 9 (Springer, Berlin, 1995).

[11] Y. G. Sinai, in Nonlinear Waves, edited by A. V. GaponovGrekhov (Nauka, Moscow, 1979), pp. 192-212.

[12] R. V. Plykin, Math. USSR Sb. 23, 233 (1974).

[13] R. F. Williams, Publications Mathématiques de L'IHÉS. 43, 169 (1974).

[14] A. Katok and B. Hasselblatt, Introduction to the Modern Theory of Dynamical Systems (Cambridge University Press, New York, 1995).

[15] V. Afraimovich and S.-B. Hsu, Lectures on Chaotic Dynamical Systems (International Press, Somerville, MA, 2003).

[16] Ya. B. Pesin, Zurich Lectures in Advanced Mathematics (European Mathematical Society, 2004).

[17] T. J. Hunt and R. S. MacKay, Nonlinearity 16, 1499 (2003).

[18] D. Ruelle and F. Takens, Commun. Math. Phys. 20, 167 (1971).

[19] M. Benedicks and L. Carleson, Ann. Math. 133, 73 (1991).
[20] C. Bonatti, L. J. Diaz, and M. Viana, in Encyclopedia of Mathematical Sciences, Vol. 102 (Springer, Berlin, 2005).

[21] V. Belykh. I. Belykh, and E. Mosekilde, Int. J. Bifurcation Chaos Appl. Sci. Eng. 15, 3567 (2005).

[22] A. A. Andronov, A. A. Vitt, and S. Ė. Khaikin, Theory of Oscillators (Pergamon Press, Oxford, 1966).

[23] S. P. Kuznetsov, Phys. Usp. 54, 119 (2011).

[24] S. P. Kuznetsov, Phys. Rev. Lett. 95, 144101 (2005).

[25] S. P. Kuznetsov and E. P. Seleznev, J. Exper. Theor. Phys. 102, 355 (2006).

[26] S. P. Kuznetsov and A. Pikovsky, Physica D 232, 87 (2007).

[27] L. V. Turukina and A. Pikovsky, Phys. Lett. A 375, 1407 (2011).

[28] S. P. Kuznetsov and I. R. Sataev, Phys. Lett. A 365, 97 (2007).

[29] D. Wilczak, SIAM J. Appl. Dyn. Syst. 9, 1263 (2010).

[30] S. Banerjee, J. A. Yorke, and C. Grebogi, Phys. Rev. Lett. 80, 3049 (1998).

[31] Z. Elhadj and J. C. Sprott, Front. Phys. China 3, 195 (2008).

[32] M. Drutarovsky and P. Galajda, in Radioelektronika, 2007 17th International Conference (Springer, Berlin, 2007), pp. 1-6.

[33] G. Benettin, L. Galgani, A. Giorgilli, and J.-M. Strelcyn, Meccanica 15, 9 (1980).

[34] Y.-C. Lai, C. Grebogi, J. A. Yorke, and I. Kan, Nonlinearity 6, 779 (1993).

[35] Y. Hirata, K. Nozaki, and T. Konishi, Prog. Theor. Phys. 102, 701 (1999).

[36] V. S. Anishchenko, A. S. Kopeikin, J. Kurths, T. E. Vadivasova, and G. I. Strelkova, Phys. Lett. A 270, 301 (2000).

[37] P. V. Kuptsov and S. P. Kuznetsov, Phys. Rev. E 80, 016205 (2009).

[38] O. B. Isaeva, A. Yu. Jalnine, and S. P. Kuznetsov, Phys. Rev. E 74, 046207 (2006).

[39] O. B. Isaeva, S. P. Kuznetsov, and A. H. Osbaldestin, Physica D 237, 873 (2008).

[40] A. Yu. Jalnine and S. P. Kuznetsov, Tech. Phys. 52, 401 (2007).

[41] A. H. Nayfeh, Perturbation Methods (Wiley, Berlin, 2000).

[42] E. Grebenikov, Yu. A. Mitropolsky, and Y. A. Ryabov, Asymptotic Methods in Resonance Analytical Dynamics (CRC Press, Boca Raton, 2004). 